\title{
Tres estilos para matar al ángel de la casa. Dar punto y final al silencio de sus voces
}

\author{
Silvia Corral Robles y Gracia González-Gijón. Universidad de Granada
}

Recepción: 30 de julio de 2016 | Revisión: 6 de septiembre de 2016 | Aceptado: 26 octubre de 2016

Correspondencia: Gracia González-Gijón | Email: graciag@ugr.es

Citar: Corral, S. y Gonzalez-Gijon, G. (2016). Tres estilos para matar al ángel de la casa. Dar punto y final al silencio de sus voces. ReiDoCrea, 5, 247-254.

\begin{abstract}
Resumen: El presente ensayo reflexiona sobre tres obras que simbolizan distintas vías de escape a la dominación patriarcal por medio del enfrentamiento de tres mujeres con este constructo social. En el "El empapelado amarillo" (1892), Charlotte Perkins Gilman, nos muestra al inicio a la protagonista como mujer adoctrinada dentro de la cultura patriarcal. Debido a este rol pasivo, su única vía de escape hacia la libertad es la locura. En Trifles (1916) (traducida como Bagatelas, cosas insignificantes), Susan Gaspell, narra su propio deseo por buscarse a sí misma como ser autónomo. A través de su obra, nos introduce en un estudio psicológico y cultural que demuestra el estatus de la mujer en la sociedad del momento, así como la concepción de los hombres hacia las mujeres como cosas insignificantes. Susan, para acabar con la situación en desventaja de la mujer, presenta a su protagonista como la asesina de su marido, y al mismo tiempo, de la opresión patriarcal. Y finalmente, la historia de Harriet Jacobs, Incidentes en la vida de una joven esclava (1861), describe la lucha incesante de una mujer negra y esclava que consigue superar todos los obstáculos de la vida a través del uso del razonamiento contra su opresor. En definitiva, estas tres obras nos van a guiar en nuestro viaje hacia el descubrimiento de esas voces, que estuvieron silenciadas.

Palabras clave: Autoras feministas | Literatura de los siglos XIX y XX
\end{abstract}

Three styles to kill the angel of the house. The silence the final point and their voices

Abstract: This essay reflects on three literary works that symbolize different escape routes from patriarchal domination through the fight of three women against this social construct. In the literary work called The Yellow Wallpaper (1892), Charlotte Perkins Gilman shows us the protagonist as a woman indoctrinated by the patriarchal culture. Due to this passive role, her unique way of escaping to freedom was madness. In Trifles (1916), Susan Glaspell describes her own desire of being a free individual. Within her work, she introduces us to a psychological and cultural study that probes the status of women in society. It also refers to men's view of women as trifles. Glaspell presents her protagonist as the murderess of her husband and at the same time, of patriarchal oppression, in order to end women's disadvantages. Finally, the story of Harriet Jacobs, Incidents in the life of a slave girl (1861), describes the eternal struggle of a black slave woman who overcomes all the obstacles in her life as a slave through the use of reasoning against her oppressor. In short, these three literary works will guide us on a journey to discover these voices that were once silenced.

Keywords: Feminist Authors | Literature of the $19^{\text {th }}$ and $20^{\text {th }}$ century

\section{Introducción}

Si hacemos un breve recorrido histórico, son muchas las escritoras que, adelantadas a su tiempo, dedicaron su vida a denunciar una dominación patriarcal abusiva a través de la literatura. Escritoras de renombre como, Mary Wollstonecraft, Sylvia Plath, Virginia Woolf, Charlotte Perkings Gilman, Susan Glaspell, Kate Chopin, Fanny Fern, Harriet Ann Jacobs, las hermanas Brönte, entre una larga lista, son consideradas hoy, autoras feministas, pese al hecho de que muchas de ellas no se considerasen a sí mismas como tal. No obstante, siendo o no conscientes del impacto que produciría el estudio de sus obras en la actualidad, sus palabras denunciaban la situación de opresión que la mujer estaba viviendo en ese momento (Snodgrass, 2006).

El contexto histórico en el que estas mujeres vivieron, siglo XIX y principios del XX, las consideraba ciudadanas de segunda clase. Éstas eran dependientes de los hombres, y la única esperanza que tenían para conseguir prosperidad económica era el matrimonio o la herencia. Pero, el matrimonio, fuera de ser una esperanza, en la gran mayoría de los casos se convertía en una esclavitud virtual que las relegaba a tareas relacionadas con la ocupación de la casa (Harris, 2008; Barker-Benfield, 2000). 
La expresión acuñada por Coventry Patmore (1854), en Thackeray, The angel in the house en su famoso poema narrativo con el mismo título publicado, era la perfecta descripción para el ideal de mujer victoriana. Se esperaba de ella sumisión y devoción y que se ajustara a la descripción estereotipada de mujer, (pasiva, débil, encantadora, elegante, dócil, tímida, sacrificada, pero sobre todo, pura), llegando a ser "el ángel de la casa". Patmore, en su poema, describía a su mujer en estos términos ya que para él eran seres creados para complacer a los hombres (Thackeray, 2011).

Por ello, cada una de las obras, que estas autoras dedicaban a esta lucha, estaban dirigidas con especial atención a las propias mujeres, víctimas de esta sumisión. Harriet Ann Jacobs en su libro, Incidentes en la vida de una joven esclava nos ofrece su visión en este sentido: "Rise up, ye women that are at ease! Hear my voice, ye careless daughters! Give ear unto my speech" ("Levantad mujeres acomodadas! Escuchad mi voz, descuidadas hermanas! Estad atentas a mis palabras!" (Clarke, 1833, p.748).

Con estas palabras pretendía promover el cambio hacia una conciencia activa contra la opresión y la sumisión del sistema patriarcal.

En esta línea, podemos decir que las autoras trataban en sus novelas, no sólo las ansiedades provocadas por el aprisionamiento y la represión que sufrían en el día a día doméstico, sino también las aberraciones que la sociedad patriarcal llevaba a cabo en detrimento de su persona, por el hecho de ser mujeres.

Una muestra de todo lo expuesto hasta aquí, aparece en las tres novelas seleccionadas en el presente trabajo. Dicha selección pretende demarcar tres posibles vías de escape, por parte de la mujer a esta opresión descrita, y por tanto, el enfrentamiento de tres mujeres con este sistema patriarcal. Las autoras elegidas son Charlotte Perkins Gilman, Susan Glaspell y Harriet Ann Jacobs.

En primer lugar, comenzaremos con el análisis de El empapelado amarillo (1892), de Charlotte Perkins Gilman; seguiremos con la obra de Susan Glaspell Ilamada Trifles (1916) (traducida como Bagatelas o cosas insignificantes); y, finalmente analizaremos la cruda historia de Harriet Jacobs, Incidentes en la vida de una joven esclava (1861).

\section{EL EMPAPELADO AMARILLO (1892), DE CHARLOTTE PERKINS GILMAN}

Más allá de resultar una historia de ficción que refleja la situación de una mujer en esa época, estamos ante un relato autobiográfico, ya que la autora sufrió el drama que se describe en esta novela corta.

Para comenzar, nos centramos en los aspectos más relevantes de la obra, con el fin de guiar al lector en el análisis. La protagonista es una mujer, cuyo nombre no aparece en la novela, (aspecto que la invisibiliza), narradora al mismo tiempo de su propia historia, que ha dado a luz recientemente y puede estar sufriendo depresión pos-parto. El doctor, quien a su vez es su marido, John (ejerciendo un doble poder frente a su mujer: doctor y marido), le ha diagnosticado una ligera tendencia histérica. De este modo, se le recomienda guardar reposo alejada de toda actividad que suponga algún esfuerzo (la lectura y la escritura parecían suponer un gran esfuerzo, además de una amenaza para sus maridos, pues la educación abre la mente y con ella, la conciencia). Esto conlleva a su "aprisionamiento" en un ático, donde sufrirá una crisis nerviosa.

Sólo hace falta la lectura de las primeras líneas de esta obra para ser testigo del proceso de adoctrinamiento que se ejerce sobre la protagonista por parte de su marido 
y que se identifica claramente en la siguiente frase: [Protagonista]: "John se ríe de mí, por supuesto, pero esto se espera en un matrimonio" (Gilman, 1892, p.647). Al leer esta frase, podemos comprobar cómo la protagonista acepta el sistema patriarcal en el que se encuentra sometida (Dock, 1996). Ella acepta todo lo que su marido dice o hace, puesto que él es la persona que sabe lo que ella tiene o debe hacer. Por ejemplo, para él es más fácil decir que presenta una "ligera" tendencia nerviosa a realmente cuestionar su problema, y por consiguiente, sus propios conocimientos en medicina.

Llegado a este punto, nuestra protagonista se encuentra encerrada en un ático, situación que va a destruir su voz como mujer, y su libertad. Tanto es así, que el propio John le da un uso específico a este ático: [John]: "Por eso pusimos la habitación del bebé en lo alto de la casa" (Gilman, 1892, p.648). Aquí, el significado de "habitación del bebé" no es literal, puesto que es la habitación de la protagonista; sin embargo, John la llama de esta forma con la intención de infantilizar a la mujer, privarla de su edad adulta, de su madurez. De hecho, en América, el concepto de ático se relaciona con una habitación destinada a los juegos de los niños (Treichler, 1984).

Asimismo, la protagonista describe la habitación como tal: "Primero era la habitación de los niños, después pasó a ser zona de juegos, y gimnasio creo; ya que las ventanas tienen barrotes para niños pequeños, y hay anillos y cosas en las paredes" (Gilman 1892, p.650). Las paredes de esta habitación, aspecto clave en la novela, estaban forradas con papel pintado de amarillo. La protagonista las describe como un "color repelente, un vivo y sucio amarillo, extrañamente descolorido por la luz del sol". "No hay duda de que los niños lo odiarían, ipero yo también lo haría si tuviese que vivir aquí por mucho tiempo!" (Gilman 1892, p.649). Este empapelado le va a crear una obsesión difícil de olvidar.

Como hemos mencionado, a la protagonista, no se le está permitido trabajar ni realizar ninguna actividad, entre ellas, la lectura y la escritura porque éstas suponen un desafío al sistema patriarcal, como modo de liberación: [Protagonista] "Así que, tomo fosfatos o fosfitos- sea lo que sea eso, y tónicos, y paseos, y aire y ejercicio, y se me está absolutamente prohibido 'trabajar' hasta que esté bien de nuevo" (Gilmam, 1892, p.648). Estas medidas prescritas por John sólo tienen la pretensión de restablecer a su esposa como ama de casa y madre (Kohzadi y Azizmohammadi, 2011).

Hay algo importante que debemos señalar en este punto, y es que, la historia que leemos, es la historia que la protagonista está escribiendo en su diario, al mismo tiempo que van sucediendo los hechos, pese a la negativa de su marido a que realice dicha tarea. [Protagonista] "Aquí viene John, y debo poner ESTO lejos, odia verme escribir una sola palabra" (Gilman, 1892, p.649). Ella sabe que lo que hace está prohibido; sin embargo, utiliza la escritura como válvula de escape.

A lo largo de la historia, la protagonista va perdiendo su capacidad para pensar razonadamente, pues todo ese tiempo que pasa alejada de la vida real y fuera de toda actividad social provoca en ella una degradación mental, hecho que puede observarse en la calidad de su escritura (Dock, 1996). Igualmente, podemos observar en sus palabras su obsesión por el papel amarillo, que se ve agudizada día tras día:

"Hay cosas en este papel que sólo conozco yo [...] Detrás de este estampado las tenues formas se ven más fácilmente cada día. Es siempre la misma forma, repetida muchas veces. Y es como una mujer agachada moviéndose lentamente detrás de este estampado. No me gusta nada" (Gilman, 1892, p.653). 
Ella proyecta sus traumas, sus más profundos sentimientos, sus obsesiones y represiones en la mujer que ve en el papel. Poco a poco se va a ver identificada con esa mujer, y el papel va a cobrar vida para ella. Lejos de ser consciente de su problema, ella expresa la dificultad que le causa hablar de este tema con John, "porque él es tan inteligente, y porque me ama tanto" (Gilman, 1892, p.653). Aquí vemos de nuevo su concepción sobre la figura del hombre, incapaz de contradecirlo o enfrentarse a él. En esta época en América, las mujeres como individuos, debían tener control sobre sí mismas y no desafiar a sus maridos, si lo hacían era porque estaban "fuera de control".

Aquí comienza su viaje hacia la locura, puesto que estas visiones en el papel ya no eran esporádicas. Cada noche con la luz de las velas, de la lámpara, con el ocaso y con la luz de la luna (un signo de feminidad) aparecían esas formas en el papel: "A veces pienso que hay muchas mujeres detrás, y a veces sólo una, y ella gatea rápido alrededor, y su gateo hace que todo tiemble" (Gilman, 1892, p.655).

La protagonista se ve cada vez más identificada con la mujer del papel. Este hecho le ha permitido que se establezca un intercambio de personalidades, aceptando de este modo, la única forma que tiene para escapar de este sistema patriarcal en el que está sometida.

Ella nunca dejaría a John, por lo que la única salida para ser libre es la locura. "Yo tiro y ella agita, yo agito y ella tira, y antes del amanecer habíamos quitado todo el papel de la pared" (Gilman, 1892, p.655). La protagonista había conseguido, por fin, deshacerse de todo el papel que le obsesionaba, ese estampado "en forma de sistema patriarcal", que la había estado silenciando durante todo este tipo. Lo había conseguido, ya era libre. Se había vuelto loca, pues era la única salida para enfrentarse a la sociedad (Martin, 1997).

\section{TRIFLES (1916) DE SUSAN GLASPELL}

La escritora Susan Glaspell sentía total dedicación hacia su marido en la vida real, sin embargo creía que necesitaba encontrarse a sí misma, buscar su autonomía. Por ello, decidió escribir una obra de teatro donde pudiese expresar ese deseo de libertad (Ozieblo, 2000).

Ésta fue una obra con un único acto, cuyo argumento giraba en torno al descubrimiento de pistas claves para resolver un asesinato por parte de las esposas de dos oficiales. Sin embargo, la obra no trata sobre un misterio de asesinato, sino más bien sobre un estudio cultural y psicológico que muestra la posición de la mujer en la sociedad del siglo XIX y XX y su intuitivo entendimiento de la realidad. Además, se añade a la temática central de la obra, la visión que tienen los hombres de las mujeres como bagatelas (tonterías, cosas de poco valor).

En esta obra de teatro, cada detalle cobra una notable importancia, pues hasta los nombres de los personajes nos indican la línea por la que la autora quiere guiar al lector. Pero antes de comenzar con el análisis de la obra es necesario situarnos en la descripción de la misma; El escenario donde se desarrolla la trama, es en una cocina de una casa rural. En la escena nos encontramos a dos oficiales buscando pistas para la resolución del asesinato del hombre de la casa, John Wright. A su vez, están allí las esposas de los oficiales buscando ropa para Minnie Wright (esposa de la víctima) encarcelada momentáneamente, al ser encontrada en el lugar del crimen. 
Como hemos mencionado anteriormente, los nombres propios tienen mucha importancia en esta obra. El nombre de la pareja del personaje asesinado, nos describe la situación de la mujer por parte de la autora, pues Minnie significa pequeño, diminuto; mientras que el apellido del marido presenta un juego de palabras: W-right, que significa correcto, adecuado.

Volviendo al escenario, en la época en la que se escribe la obra, la cocina era el reflejo de la personalidad de la mujer; si la cocina estaba desordenada (como ocurre en este relato), la mujer suponía un peligro para la sociedad. Sin embargo, los oficiales relacionan lo observado en la cocina como "tonterías de mujeres": [Sheriff: "Aquí no hay nada sólo cosas de cocina" (Glaspell, 1916, p.982). Por su parte, las mujeres de los oficiales deciden "ayudar" a la investigación buscando pistas a su alrededor. Sin embargo, como mujeres de los oficiales deben ser fieles a la ley y a sus maridos, aunque ambas conocen a John Wright y el tipo de marido que era (Radner y Lanser, 1987).

Las dos mujeres comentan como es Minnie Wright: "Ella solía llevar bonitas ropas y era muy alegre, cuando era Minnie Foster era una de las mujeres que cantaba en el coro de la ciudad. Pero esto- oh, hace treinta años de eso" (Glaspell, 1916, p.984). De este último comentario, deducimos un hecho que nos conduce a recordar el famoso mito de Procne y Filomena. A ésta última, le cortaron la lengua con el fin de no desvelar la violación que había sufrido por parte de Tereo, marido de su hermana Procne. Tanto Minnie como Filomena fueron despojadas de su derecho a hablar al ser forzadas a una posición de subordinación ante el sistema patriarcal.

Como hemos descrito anteriormente, las mujeres encuentran entre las tonterías de la cocina pistas clave para el caso, entre ellas, una pieza de una colcha que estaba haciendo la señora Wright y una jaula con la puerta rota, como si alguien hubiese mostrado violencia al tirar de ella. Al observar la colcha descubren que los últimos puntos dados en ella están alterados, transformándose el tejido y los puntos dados en él, en un modo de escritura, donde se puede expresar no sólo la creatividad sino también los temores, sentimientos e inquietudes. Sobre este hecho, en la obra aparece la palabra inglesa "knot" para hablar de 'nudo', pero además, esta palabra también significa "casarse", apareciendo de nuevo un juego de palabras, cuya relación de significados tendrá coherencia al final de la obra.

En relación a la jaula, las señoras no entendían por qué estaba vacía y con ese aparente signo de violencia. En este punto, se relaciona a la señora Wright y su gusto por la canción, con un pájaro: [Una de las señoras] "Ella solía cantar muy bien" (Glaspell, 1916, p.986). No obstante, la verdad se descubre poco después, cuando encuentran un pequeño canario muerto escondido en otra pieza de colcha. [Una de las señoras]: "Alguien retorció su cuello" (Glaspell, 1916, p.987), recordando al señor Wright, quién también fue estrangulado.

Sin apenas apreciarlo, este fue el momento en que las señoras descubrieron a la asesina del señor Wright, Minnie Wright. [Una de las señoras]: "No, al señor Wright no le gustaba el pájaro, una cosa que cantaba. Ella solía cantar también. El mató todo eso" (Glaspell, 1916, p.988). El señor había matado su canario, acto simbólico de lo que él ya le había hecho a ella: había destruido su voz como mujer (Ozieblo, 2000).

Como afirma Ben-zvi (1992), las mujeres que matan producen miedo porque ellas están desafiando los constructos sociales de feminidad, pasividad, contención y educación. No obstante, las mujeres de los oficiales aun sabiendo todo lo ocurrido y estando "casadas con la ley", esconden sus pruebas, consideradas por sus esposos 
como "tonterías de mujeres". Para ellos, era más fácil pensar que alguien del exterior había cometido el asesinato puesto que veían a Minnie como una mujer débil, no lo suficientemente fuerte para llevar a cabo este homicidio. El hecho de reconocer la validez de las evidencias que las mujeres encontraron, ignoradas anteriormente, supondría el reconocimiento de las "peligrosas" cualidades de una mujer, dejando de ser frágiles criaturas y suponiendo así, el deseado cambio de la sociedad.

Susan Glaspell, adelantada a su tiempo, describe en esta obra el maltrato emocional y la violencia simbólica a la que las mujeres son sometidas. Aunque no podemos juzgar al señor Wright de haber cometido maltrato físico a su mujer, sin embargo, el hecho de agredir o dañar a su mascota puede incluir este tipo de maltrato. En la actualidad, muchas mujeres siguen sufriendo esta opresión del sistema patriarcal que las silencia y las ahoga por dentro un día tras otro (Ben-Zvi, 1992).

\section{INCIDENTES EN LA VIDA DE UNA JOVEN ESCLAVA (1861) DE HARRIET ANN JACOBS}

Esta obra, que se desarrolla en el contexto de la esclavitud en América, sigue la misma línea de análisis que hemos trazado a lo largo del trabajo: la posición de la mujer, y los medios para escapar de ese sistema patriarcal en el que se encuentran inmersas.

La protagonista de esta obra ha sufrido el maltrato por ser mujer, mujer negra y esclava; no permitiéndole tener una vida mínimamente digna. Su mundo exterior le resultaba una enorme prisión, y su mundo interior reflejaba esa realidad. Harriet Ann Jacobs, es la autora de este libro que narra, a través del pseudónimo, Linda Brent, la vida que le fue otorgada: "nací siendo esclava" (Jacobs, 1861, p.5). Con esta frase comienza su desgarradora historia como esclava a través de la cual pretende reivindicar el sentimiento de hermandad entre las mujeres, así como la conciencia ante esta situación, haciendo uso de un lenguaje crudo y realista.

En el capítulo llamado El periodo de prueba hacia la juventud, Harriet, a la edad de quince años, narra cómo su patrón le susurraba al oído sucias palabras. Sin embargo, ante su rechazo él le recordaba que "yo era de su propiedad" "y que iba a conseguir todo lo que desease" (Jacobs, 1861, p.27). Este período de edad era muy diferente para las chicas blancas que para las de color. Para las primeras significaba la búsqueda de un buen marido, la iniciación en el sexo y la ciencia doméstica (ser una perfecta ama de casa), mientras que para las chicas de color todo era diferente. Ellas no merecían ser llamadas "ladies" y podían ser violadas sin que nadie fuese a denunciar la situación (Cutter, 1996). Esto sucede en su caso, pues los principios de pureza inculcados por su abuela fueron corrompidos finalmente por este hombre, quedando así embarazada.

Ante esta situación, Harriet, tramó un plan, pues si no lo hacía, su hijo iba a pasar a ser propiedad de ese viejo tirano. Ésta, buscó un hombre blanco que no estuviese casado y con una buena posición que le permitiese cuidar a su hijo como una persona libre. Ella afirma en su libro que "yo sabía lo que había hecho y lo hice con un cálculo deliberado" (Jacobs, 1861, p.54). Harriet no era una víctima pasiva y no pretendía convertirse en el juguete de su patrón. Sin embargo, su plan salió mal y finalmente este hombre blanco que la iba a ayudar, pretendía dar su hijo a los esclavos de una mujer blanca para llevárselo al norte. Por lo que nuestra protagonista volvió a estar en manos del patrón. 
Pese a estar de vuelta en su destino fijado, Harriet nunca se dio por vencida y día tras día luchaba para no ser la víctima. Todo lo contrario que en la novela de Charlotte Perkins Gilman, en el que la protagonista es aprisionada en el ático, Harriet se recluye a sí misma con el fin de alcanzar dos objetivos: uno, cumplir su penitencia, pues en la comunidad cristiana es la única forma de limpiar tus pecados; y el segundo, el empleo de la escritura para destruir al patrón. Harriet, durante los siete años que va a permanecer aprisionada, va a manipular unas cartas que le envía al patrón para confundirlo y volverle loco, destruyéndolo y liberándose así misma y a la voz que le fue silenciada al nacer (Cutter, 1996).

Harriet al escribir su libro afirma que se está quitando "la mordaza de hierro" impuesta por la esclavitud para usar así su voz y denunciar lo que estaba sucediendo con las mujeres.

\section{Conclusiones}

Para concluir, quisiéramos recordar el título que da nombre a nuestro trabajo: Tres estilos para matar el ángel de la casa. Dar punto y final al silencio de sus voces. Nuestras protagonistas, tres mujeres encadenadas dentro de un sistema patriarcal organizado desde principios de los tiempos, con historias y experiencias diferentes han puesto punto y final a un silencio impuesto, han "matado" ese ángel que las etiquetaba y las restringía de toda condición social o pública por el mero hecho de ser mujeres. Cada una ha encontrado su forma de actuación para lograr su meta configurando así diferentes estilos.

En el caso de la literatura negra, hemos observado como las mujeres luchan en contra de las adversidades, utilizando el razonamiento contra su opresor. Al igual, Minnie, de Bagatelas, desafió a su marido no dejando indiferente a nadie. Tanto fue así, que esta obra no fue bien recibida por las mujeres de la época, la cual fue anterior al movimiento de los derechos de las mujeres. Por último, la protagonista sin nombre de El empapelado amarillo encerrada bajo llave en una habitación destinada para niños, se vio obligada a escapar del sistema en detrimento de su persona.

Pese a las diferencias encontradas en las obras, las tres protagonistas comparten el sufrimiento de la violencia que ejerce el patriarcado contra ellas. En los tres casos, éstas han sido privadas de sus voces, de su propio ser (Ozieblo, 2000). Sin embargo, "desafiando la idea de debilidad" impuesta en las mujeres por la sociedad patriarcal, las tres protagonistas han demostrado su atrevimiento al oponerse a un sistema que las ha sublevado e infravalorado.

El deseo de búsqueda de una nueva forma de vida dónde toda desigualdad quedase atrás fue la reivindicación que estas autoras pretendieron realizar a través de la literatura; lamentablemente siglos después la situación no dista mucho de lo descrito en estas tres obras. Seguimos sin superar la distancia que nos separa de los derechos de los hombres, aunque tengamos la ilusión de que si lo hemos conseguido. Como mujeres nos queda un largo camino por recorrer, pero si algo hemos aprendido con las historias de nuestras protagonistas es que debemos aplaudir el modelo de mujer que se atreve a dar punto y final... para escuchar más fuerte que nunca su voz silenciada.

Referencias 
Ben-Zvi, L. (1992). Murder, she wrote: The Genesis of Susan Glaspell's Trifles. Theatre Journal, 44(2), 141-162.

Clarke, A. (1833). The holy Bible, containing the Old and New Testaments: The text printed from the most correct copies of the present authorized translation including the marginal reading and parallel texts, with a commentary and critical notes. Designed as a help to abetter understanding of the sacred writings. New York: Royal octabo Stereotype Edition.

Cutter, M. (1996). Dismantling The Master's House: Critical Literacy in Harriet Jacobs. Incidents in the Life of a Slave Girl. Callaloo,19(1), 209-225.

Dock, JB. (1996). But One Expects That: Charlotte Perkins Gilman's 'the Yellow Wallpaper' and the Shifting Light of Scholarship. Publications of the Modern Language Association of America,111(1), 52-65.

Glasspell, S. (1916). Trifles. http://www.english.unt.edu/ simpkins/Trifles.pdf

Harris, JE. (2008). The impact of gender socialization on women's learned technological helplessness and its anagogical implications. Tesis Doctoral, Graduate School of the University of Missouri.

Jacobs, H. (1894). Incidents in the Life of a Slave Girl Written by Herself. London, England: Harvard University Press.

Kohzadi, H., \& Azizmohammadi, F. (2011). An Analysis on Material Feminism. Australian Journal of Basic and Applied Sciences, 5(12), 22462251.

Martín, S. (1997). La loca en el desván: Consecuencias psicológicas de la violencia patriarcal en algunas novelas del siglo XIX. Asparkía. Investigació feminista, 8, 89-102.

Ozieblo, B. (2000). Para ser libre. La angustia de la violencia en las obras de Susan Glaspell y Sophie Treadwell. Dossiers feminists, 4, 115-129.

Gilman, C. (1892). The Yellow Wallpaper. https://www.nlm.nih.gov/literatureofprescription/exhibitionAssets/digitalDocs/The-Yellow-Wall-Paper.pdf

Radner, J., \& Lanser, S. (1987). The Feminist Voice: Strategies of Coding in Folklore and Literature. The Journal of American Folklorev, 100(398), 412-425.

Snodgrass, M. (2006). Encyclopedia of Feminist Literature. Nueva York: Facts On File.

Thackeray, W. (2011). The angel in the house. http://academic.brooklyn.cuny.edu/english/melani/novel_19c/thackeray/angel.html

Treichler, P. (1984). Escaping the Sentence: Diagnosis and Discourse in 'The Yellow Wallpaper. Tulsa Studies in Women's Literature. 3(2), 61-77. 